\title{
Dielectric properties of ternary composites of triblock polymer surfactant, liquid crystal and organic solvent
}

\author{
M. R. Fisch ${ }^{*}{ }^{\S}$, Clinton Braganza ${ }^{\dagger}$, L. C. Chien ${ }^{\dagger}$ and R. G. Petschek ${ }^{\ddagger}$ \\ ${ }^{*}$ College of Applied Engineering, Sustainability, and Technology \\ Kent State University, Kent, Ohio 44242, USA \\ ${ }^{\dagger}$ Liquid Crystal Institute and Chemical Physics Interdisciplinary Program \\ Kent State University, Kent, Ohio 44242, USA \\ tDepartment of Physics, Case Western Reserve University, 10600 Euclid Ave. \\ Cleveland, Ohio 44106, USA \\ $\S_{\text {mfisch@kent.edu }}$
}

Received 29 May 2014; Revised 14 July 2014; Accepted 18 July 2014; Published 3 September 2014

\begin{abstract}
Dielectric spectroscopy, at room temperature $\left(20^{\circ} \mathrm{C}\right)$, is used to study the dielectric response of ternary mixtures of commercial nematic liquid crystal mixtures E7 and E33, an organic solvent N-Methyl-2-Pyrrolidone (NMP) and a triblock polymers in the frequency range from $0.01 \mathrm{~Hz}$ to $1 \mathrm{MHz}$. The results indicate a dielectric relaxation in the hectohertz region. Individually, both E7 and NMP have rather large low frequency conductivities; however, the low frequency $(0.01-10 \mathrm{~Hz})$ behavior of the mixtures has no such behavior. We attribute this behavior to an ion getter effect of the triblock polymer surfactant. Optimized ternary mixtures obtain a real dielectric constant near 230, and loss tangent less than 0.05 at frequencies near $10 \mathrm{mHz}$.
\end{abstract}

Keywords: Emulsion; dielectric properties; liquid crystal.

\section{Introduction and Motivation for Study}

There is continuing interest in new electrical energy storage devices; such devices are characterized by both energy density and power density. ${ }^{1}$ Energy density is the energy per unit mass or volume and power density is the power per unit mass or volume. In a linear dielectric, the energy density $u$ is given by:

$$
u=\frac{1}{2} \varepsilon E^{2},
$$

where $\varepsilon$ is the dielectric constant and $E$ the electric field. Thus, to increase the energy density one must either increase the dielectric constant and/or increase the maximum (breakdown) electric field.

There has been significant research into increasing the dielectric constant through the use of polymer composites. For example, commercially available Biaxially Oriented PolyPropylene (BOPP)-based capacitors ${ }^{2}$ obtain energy densities $u \approx 1.2 \mathrm{Jcm}^{-3}$ through a small dielectric constant (about 2.2) and a rather large breakdown electric field (650V/micron). Ongoing research with high dipole density polymers such as poly(vinylidenefluoride) (PVDF) and Trifluouroethlene (TrFE) provide for higher energy densities of about $16 \mathrm{Jcm}^{-3} .^{3}$ The dielectric constant of these polymers can be enhanced ( $\varepsilon \approx 60$ ) by making composites containing high dielectric constant ferroelectric ceramic powders such as $\mathrm{PbTiO}_{3},{ }^{4,5}$ but lead is a potentially harmful metal and inhomogeneous composites can have significantly smaller energy densities because the breakdown field may be reached locally even though globally the field is much smaller. Nickel ${ }^{6}$ or Polyaniline (PANI) ${ }^{7}$-doped PVDF composites near the percolation threshold, where many conductive particles are isolated by thin dielectrics to form microcapacitors, show an overall high dielectric constant of $\varepsilon \approx 400-1000$. The disadvantage of these systems is the low breakdown fields near the percolation threshold.

This paper presents a new materials approach of using liquid crystals, high dielectric constant liquids and surfactants to form self-assembled composite structures that have a high dielectric constant and low loss at low frequencies. Our goal is to predicatively understand, develop, and characterize these organic-organic composites and emulsions with very low electrical conductivities and large specific capacitances. Initial estimates suggest a one to three orders of magnitude or greater maximum energy density than present materials may be possible. Moreover, because these are fluid or gel phases such dielectrics will be self-healing.

These materials are based on the Maxwell-Wagner effect $^{8-10}$ that indicates that one may obtain large dielectric coefficients through interfacial polarizations; however, this comes at the cost of characteristic frequency relaxations. We show that using liquid crystals, appropriate surfactants, and solvents, it is possible to use this effect to achieve a high dielectric constant low loss (conductivity) dielectric.

This is an Open Access article published by World Scientific Publishing Company. It is distributed under the terms of the Creative Commons Attribution 3.0 (CC-BY) License. Further distribution of this work is permitted, provided the original work is properly cited. 
This paper will provide a brief review of the dielectric properties of heterogeneous systems, experimental measurement equipment, techniques and data analysis, the dielectric properties of single component systems used in the mixtures, and finally the dielectric properties of dispersed ternary systems.

\section{Theoretical Background}

The study of heterogeneous dielectrics has a long history. A recent review paper by Asami ${ }^{11}$ describes the current state-ofthe-art and little can be added. We will only summarize a few of the salient features pertinent to the present study.

Most materials do not exhibit the simple Debye behavior and more generally there is a distribution of relaxation times. A generalization, the Havriliak-Negami ${ }^{12}$ theoretical form, is useful and fits a broad range of experimental data in many materials. It is a generalization of the Debye relaxation and several of its generalizations. ${ }^{13}$ The complex dielectric constant as a function of frequency for a dielectric modeled by this theoretical form is given by:

$$
\varepsilon^{*}(\omega)=\varepsilon_{\infty}+\frac{\left(\varepsilon_{0}-\varepsilon_{\infty}\right)}{\left(1+\left(j \omega \tau_{0}\right)^{\mu}\right)^{\phi}}-j \frac{\sigma}{\omega},
$$

where $\tau_{0}$ is the characteristic relaxation time and $\mu$ and $\phi$ are empirically determined constants for a given material combination. In this expression, $\varepsilon^{*}$ is the complex dielectric constant, $\varepsilon^{*}=\varepsilon^{\prime}+j \varepsilon^{\prime \prime}$ where $j=\sqrt{-1}, \varepsilon^{\prime}$ is the real dielectric constant and $\varepsilon^{\prime \prime}$ is the imaginary part of the dielectric constant, $\varepsilon_{\infty}$ is the real high frequency dielectric constant, $\varepsilon_{0}-\varepsilon_{\infty}$ is the magnitude of the dielectric relaxation, $\omega$ is the angular frequency ( $2 \pi f)$, and $\sigma$ the conductivity of the system.

\section{Experimental Details}

The impedance of all samples was measured in lab constructed capillary filled cells whose conductors were of known separation, area and surface resistivity. Briefly, these cells were made of two pieces of soda lime float glass coated with a transparent conductive layer of Indium Tin Oxide (ITO), and a polyimide alignment layer separated by spacers. The spacing between the plates was controlled using cylindrical-shaped glass spacers of know diameter and checked using interference fringes. The electrode area was controlled by etching a pattern on the layer of ITO using standard photolithography techniques. The manufacturer of the ITO glass placed a barrier layer made of silicon dioxide between the glass and ITO layers to prevent ion diffusion from the glass. After patterning the ITO, a thin layer of polyamic acid (PAA) dissolved in N-Methyl Pyrrolidinone (NMP) was spin coated then hard baked (or imidized) to form a layer of polyimide. When rubbed in a single direction with a velvet cloth this layer defines the alignment direction for the liquid crystal in the cells. Transparent electrodes were used so that alignment and defects of the liquid crystal could be visually observed under a light microscope.

The sample's alignment was examined both before and after experimentation, and no difference observed. Aligned liquid crystal samples have well-studied microscopic textures, in which defects and inhomogeneities are clearly visible. The samples reported had textures consistent with well-aligned microscopically homogeneous liquid crystalsolvent mixtures

Since aligned liquid crystals tend to make inhomogeneities more visible, ${ }^{14}$ a typical sample was no more than $10 \mu \mathrm{m}$ thick, and liquid crystal ordering such as observed in pure liquid crystals was observed, no further tests for inhomogeneities was made. The sample details are available in Braganza's dissertation. ${ }^{15}$ The paucity of textures and optical variations are a clear indication of the sample's structural homogeneity.

The impedance was measured using a Schulmberger ${ }^{\circledR}$ (Solarton) SI 1260 impedance/gain-phase analyzer equipped with the Solartron ${ }^{\circledR}$ Dielectric interface, SI 1296 that extends the impedance analyzer's current measurement capability downwards from $6 \mathrm{~mA}$ to $100 \mathrm{fA}$ and increases the maximum allowed probe voltage from $3 \mathrm{~V}$ to $7 \mathrm{~V}$. The SI 1260 is capable of testing devices over a frequency range of $10 \mu \mathrm{Hz}$ to $32 \mathrm{MHz}$. The present experiments were conducted over a range of $10 \mathrm{mHz}$ to $1 \mathrm{MHz}$. The peak voltage for all measurements was less than or equal to 0.1 VAC. Further details about the cells and the experimental procedure are available elsewhere. ${ }^{15}$

The impedance of both the empty and the filled cells was measured. Taking the ratio of the empty impedance to the filled impedance leads to the apparent dielectric response of the liquid crystal mixtures without any lead resistance or other frequency-dependent cell properties as shown in Eq. (3).

$$
\varepsilon_{\text {full }}^{*}=\varepsilon_{\text {empty }}^{*} \frac{Z_{\text {empty }}}{Z_{\text {full }}},
$$

where $Z_{\text {empty }}$ and $Z_{\text {full }}$ are the measured complex impedance of the empty and filled cell, respectively, and $\varepsilon_{\text {full }}^{*}$ and $\varepsilon_{\text {empty }}^{*}$ are the respective complex dielectric constants.

While this does not correct for fringe fields it does approximately correct for frequency-dependent cell parameters. Since the ratio of the cell spacing to an electrode dimension is about $1000: 1$, this is expected to be a very small effect. Nevertheless, because this is not an absolute measurement, the dielectric coefficients obtained in this study are called the apparent dielectric response in this paper. When the cell was assumed to be ideal, ${ }^{\text {a }}$ the real dielectric constant at frequencies near $1 \mathrm{MHz}$ became less than 1 , a physical impossibility that has been reported.

The liquid crystal mixtures E7 and later E33 are room temperature nematic mixtures obtained from British Drug 
House (BDH). These nematic mixtures lack formal names beyond "liquid crystal mixture E\#," the CAS number for nematic mixture E7 is 63748-28-7. These materials have essentially identical material and electrical properties. They were used without further purification. The NMP (1-Methyl2-pyrrolidione) and a related material NMF (N-Methylformamide) were Fisher chromatographic grade and used as supplied. Finally the block polymers and surfactants used were obtained from Aldrich Chemical and all are solid powders that dissolve in polar solvents. ITO coated glass was supplied by Colorado Concepts Coatings and had a sheet resistance of $100 \Omega /$ sq. Except for the study of the pure liquid crystal where two different alignment directions parallel and perpendicular to the glass substrate were used; all samples were studied in cells with alignment layers (described above) that caused the pure liquid crystal to align parallel to the glass substrate.

\section{Results}

\subsection{Introduction}

Approximately 350 distinct combinations and concentrations of surfactants, liquid crystal, and solvent were studied. Many mixtures exhibited a large imaginary dielectric coefficient at frequencies below $100 \mathrm{~Hz}$. These mixtures are not discussed in the present paper. Details about their behavior are available in Braganza's dissertation. ${ }^{15}$

\subsection{Pure compounds}

To put the mixture's behavior in proper context, we will first present the results obtained for the pure E-7 and NMP. A good dielectric should have a large real dielectric constant and a small imaginary dielectric constant. At low frequencies, the two liquid components: E-7, the nematic liquid crystal, and NMP are poor dielectrics. However, they do exhibit a large real dielectric constant that will be harnessed in the mixtures.

The results obtained for the liquid crystal are shown in Fig. 1. Notice that dielectric properties depend on the alignment of the molecules as expected, and the imaginary part of the dielectric constant is comparable to the real part at low frequencies $(f<1 \mathrm{~Hz})$ in a manner that is indicative of a strong contribution from the conductivity of the material. This suggests, as is well known, that nematic liquid crystals are poor insulators at low (near DC) frequencies. It also explains why the addressing frequency of displays using this liquid crystal are near $1 \mathrm{kHz}$ since near this frequency the imaginary dielectric constant obtains its smallest value while the real part of the dielectric constant is largely frequency independent from 50 to $20,000 \mathrm{~Hz}$ obtaining a value near 20 .

The results for the solvent NMP (1-Methyl-2-pyrrolidione) and a related material NMF (N-Methylformamide) are shown in Fig. 2.
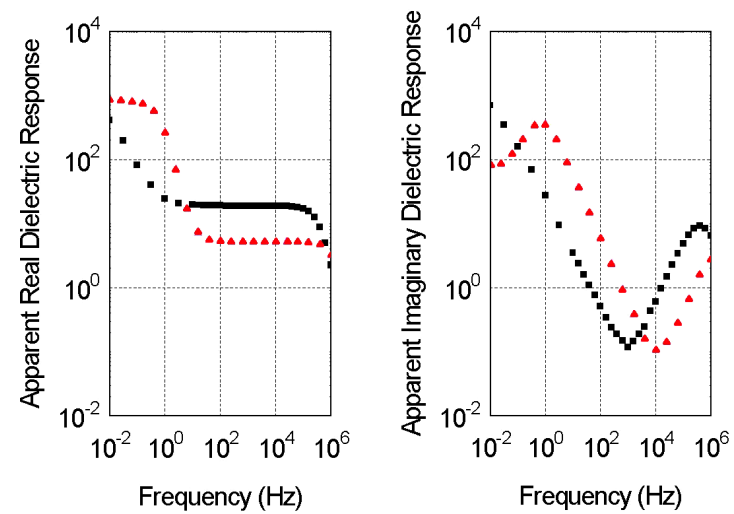

Fig. 1. The apparent dielectric response of the liquid crystal E7 as measured using cells that align its long axis either parallel to the substrate (triangles) or perpendicular to the substrate (squares).
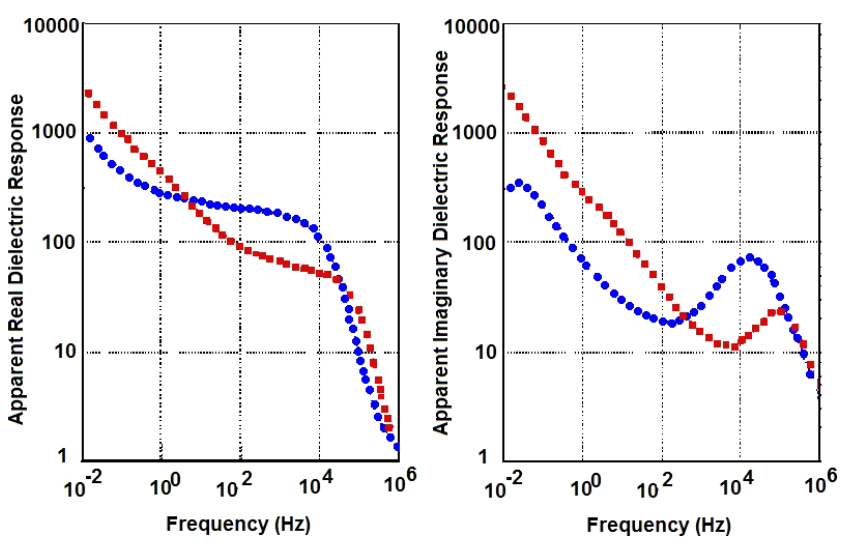

Fig. 2. (Color online) The apparent dielectric response of the organic solvents used as components in the mixtures studied. The solvents are NMP (blue and square symbols) and NMF (red and circle symbols). The real response as a function of frequency is shown on the left while the imaginary is shown on the right.

Once more notice that at frequencies below $100 \mathrm{~Hz}$ the real and imaginary part of the dielectric constant are of very similar magnitude; thus, indicating a poor dielectric. Both NMF and NMP were used in preliminary studies discussed elsewhere. ${ }^{15}$ These experiments indicated that NMP was the better solvent for the mixtures discussed in this paper because of its larger real response and smaller imaginary response. The increase in both real and imaginary dielectric response is due to conductivity presumably due to polar impurities in the solvents.

Triblock polymeric surfactants made of blocks of hydrophilic poly(ethylene glycol) and hydrophobic poly(propylene glycol) were the third component in the mixtures. These surfactants were separated into two classes depending on the position of the hydrophilic block. The first class, PEP describes the polymer in which the poly(ethylene glycol) block is positioned in the center and flanked by two hydrophobic poly(propylene glycol) blocks. The second class, EPE occurs when the poly(ethylene glycol) block flanks a central 


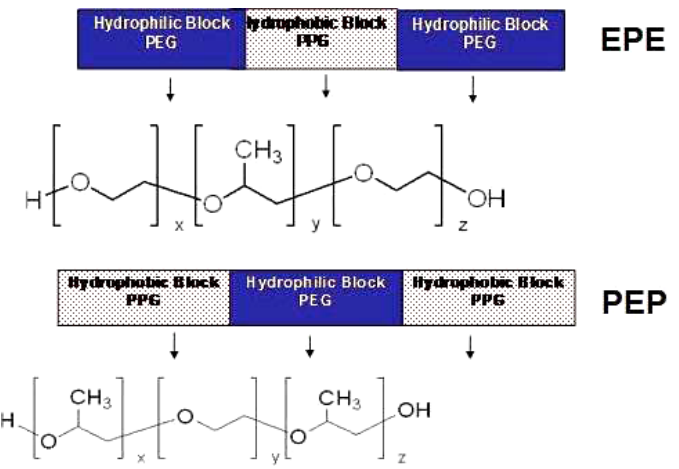

Fig. 3. Schematic figures of the triblock polymers used in this study.

hydrophobic poly(propylene glycol) block. Diblock versions of these molecules were not available for testing. Schematic figures of these surfactants are shown in Fig. 3.

The triblock polymeric surfactants studied and our naming convention is shown in Table 1 . In this table, $M_{n}$ is the number of average molecular weight ${ }^{\mathrm{b}}$ of the polymer, and HLB is Hydrophilic-Lipophilic Balance. The HLB is used to classify a surfactant according to the proportion of the hydrophilic part. Although not an exact measure, it is based on a calculation of the ratio of the molecular weight of the hydrophobic portion to the total molecular weight of the surfactant. ${ }^{17,18}$

Once more these materials cannot be classified as particularly good dielectrics since the imaginary and real dielectric constants are separated by at most about a factor of 10 at low frequencies $(f \leq 1 \mathrm{~Hz}$ ). However, the real dielectric coefficient obtains a value near 100 and a relaxation near $100 \mathrm{~Hz}$ is observed.

\subsection{Ternary mixtures}

\subsubsection{Theoretical models}

A large number of samples were investigated. The investigations included different triblock polymeric surfactants at a number of different concentrations in a solvent composed of a 10:1 mixture of E-7 and NMP. Similar investigations with NMP replaced by NMF, and fixed surfactant concentration and various concentrations of organic solvent were also conducted. A synergistic effect occurred so cell behavior

Table 1 . The copolymer series studied.

\begin{tabular}{lccccccc}
\hline & EPE0 & EPE1 & EPE2 & EPE3 & EPE4 & PEP0 & PEP1 \\
\hline$M_{n}$ & 2800 & 5800 & 2900 & 1900 & 8400 & 3300 & 2000 \\
PEG(\%) & 10 & 30 & 40 & 50 & 80 & 10 & 50 \\
HLB & $1-7$ & $7-9$ & $12-18$ & $18-23$ & $>24$ & $2-7$ & $12-18$ \\
\hline
\end{tabular}

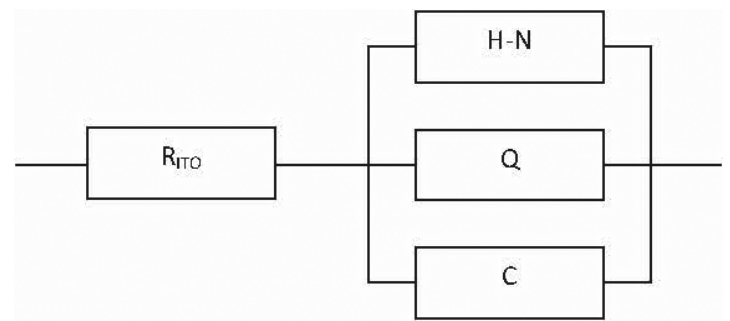

Fig. 4. The circuit used to model the apparent dielectric response measurements of mixtures. $\mathrm{H}-\mathrm{N}$ is the Havriliak-Negami element; $\mathrm{Q}$ is a constant phase element and $\mathrm{C}$ an ideal capacitor.

could be modeled as that of a Havriliak-Negami element, an ideal capacitance, a constant phase element and a series resistance due to the conductivity of the electrodes. The model used to fit the data is shown in Fig. 4.

The Havriliak-Negami model of the frequency-dependent dielectric is discussed above. It is given by Eq. (2). This equation reduces to several common expressions in appropriate limits (see footnote b), and is used to model the emulsion's dielectric properties. This model has a characteristic relaxation time, $\tau_{0}$. We report our results in terms of $f_{0}=\tau_{0}^{-1}$.

A constant phase element (CPE) is the second generic feature of our dielectric model. This element is modeled by an element that has an impedance given by: $Z_{\mathrm{CPE}}=Q(j \omega)^{-n}$ where both $Q$ and $n$ are frequency-independent terms. ${ }^{13}$ The phase angle of the CPE is frequency independent and has a value of $-90 n^{\circ}$, hence the name CPE. Generally $n \neq 0.5$, since when $n=0.5$ this corresponds to an infinite Warburg element. While it is rarely used in the following limits, it should be noted that $n=1$ corresponds to an ideal capacitor, and $n=0$ corresponds to an ideal resistor. Due to this fact it has been also named "recap" by some workers. When $n$ is near 1 , this element does a good job modeling conductivity effects. It is often used to model surface roughness effects of the electrodes. ${ }^{19,20}$

The term $\mathrm{C}$ accounts for the frequency dependence of the cells and other irregularities. An empty cell exhibits some conductance effects, stray capacitive coupling and other effects that prevent it from behaving like a simple completely isolated, ideal, point circuit element parallel plate capacitor. As stated earlier each cell was characterized before it was filled and the results of this measurement used to determine the parameters describing $C$. Since these were not free parameters in the fitting they were not adjusted, and are not reported. The term $R_{\text {ITO }}$ is used to model the resistance of the wires and the ITO in the capacitors.

\subsubsection{Experimental results: Surfactant type and concentration, NMP concentration}

The effect of all of the above triblock surfactants on the dielectric response when the solvent is $90.9 \% \mathrm{E}-7$ and 9.1\% MNP

\footnotetext{
bPolymers are in part characterized by the number of monomers in the molecule. Commercial synthetic polymers consist of molecules of varying number of monomers. This is characterized through various molecular weight averages (see for instance Ref. 16).
} 
Table 2. The fits to the data in Fig. 5, solid line showing the effect of increasing the HLB on samples containing $2 \%$ surfactant and liquid crystal E7.

\begin{tabular}{|c|c|c|c|c|c|c|c|c|c|c|}
\hline \multirow[b]{2}{*}{ Surfactant name } & \multirow[b]{2}{*}{ PEG $(\%)$} & \multirow[b]{2}{*}{ HLB } & \multirow[b]{2}{*}{$\mathrm{R}(\Omega)$} & \multicolumn{4}{|c|}{ Havriliak-Negami parameters } & \multicolumn{2}{|c|}{ CPE } & \multirow[b]{2}{*}{$\varepsilon_{\infty}$} \\
\hline & & & & $\varepsilon_{0}-\varepsilon_{\infty}$ & $f_{o}(\mathrm{~Hz})$ & $\mu$ & $\phi$ & $\mathrm{Q}$ & $\mathrm{n}$ & \\
\hline EPE0 & 10 & $1-7$ & 24.64 & 173.6 & 174.36 & 1 & 1 & 30 & 0.89 & 11.6 \\
\hline EPE1 & 30 & $7-9$ & 26.38 & 190 & 117.46 & 1 & 1 & 43 & 0.86 & 10.7 \\
\hline EPE2 & 40 & $12-18$ & 25.40 & 178 & 77.79 & 0.95 & 1 & 22 & 0.90 & 12 \\
\hline EPE3 & 50 & $18-23$ & 24.76 & 217 & 90.74 & 0.93 & 1 & 31 & 0.86 & 11.6 \\
\hline EPE4 & 80 & $>24$ & 28.62 & 208 & 115.41 & 0.95 & 1 & 24 & 0.9 & 10.9 \\
\hline PEP0 & 10 & $2-7$ & 22.59 & 171.2 & 122.99 & 0.99 & 1 & 30 & 0.89 & 11 \\
\hline PEP1 & 50 & $12-18$ & 21.61 & 168 & 122.71 & 1 & 1 & 27 & 0.9 & 11.4 \\
\hline
\end{tabular}

are remarkably similar. At a concentration of $1 \%$ polymeric surfactant, all obtain a peak in the imaginary dielectric coefficient of approximately 100 at a frequency of approximately $100 \mathrm{~Hz}$ and obtain a real dielectric coefficient that increases about $10-20 \%$ over the frequency range 100 to $0.01 \mathrm{~Hz}$ obtaining a value of approximately 230 at $f=0.01 \mathrm{~Hz}$.

Increasing the concentration of the surfactant EPE1 from $1 \%$ to $3 \%$ while keeping the fluid as $90.9 \% \mathrm{E} 7,9.1 \% \mathrm{NMP}$ has little effect $\varepsilon_{\infty}$, changing from 11.6 to 10.2 , while $\varepsilon_{0}-\varepsilon_{\infty}$ changes from 217 to 209 . However, the relaxation frequency increased from 91 to $261 \mathrm{~Hz}$. Changing the concentration of EPE2 from $1 \%$ to $3 \%$ illustrated somewhat different behavior. Here, $\varepsilon_{0}-\varepsilon_{\infty}$ obtained a minimum at $2 \%$ surfactant concentration. At the same concentration $f_{0}$ obtains a maximum of $142 \mathrm{~Hz}$ and $\varepsilon_{\infty}$ a value of 11 . Thus, the concentration of surfactant had little effect on the overall response, maximizing the effect near $2 \%$ surfactant.

Finally, changing the concentration of NMP in samples of $2 \%$ PEP showed that conductivity was a problem at high concentrations of NMP and the optimal concentration of NMP in E7 was $9.1 \%$.

To summarize this series of experiments, a surfactant concentration near $2 \%$ leads to minimal conductivity effects
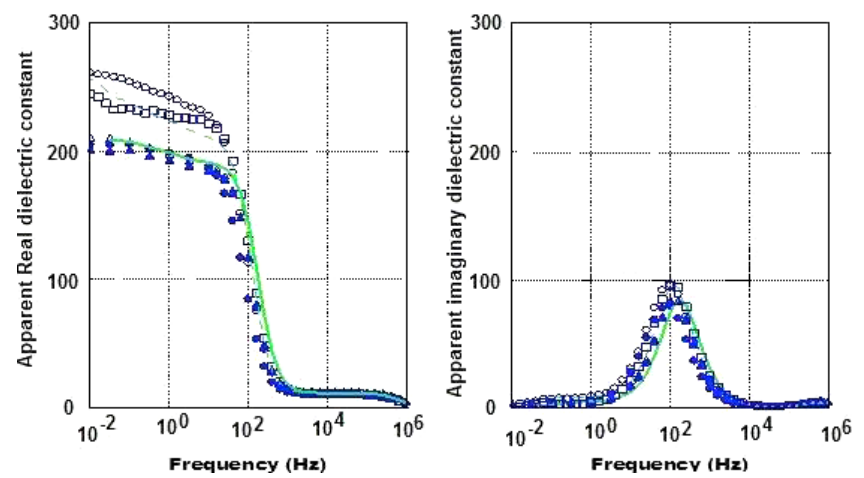

Fig. 5. The effect of increasing the portion of the PEG block in the copolymer on the apparent dielectric response of a mixture made of $1 \%$ surfactant, 9.1\% NMP and 90.9\% E7 (• EPE0, 。 EPE1, $\square$ EPE2, $\triangle \mathrm{EPE} 3, \boldsymbol{\Delta} \mathrm{EPE} 4$, broken line PEP0, solid line PEP1). Note that all surfactants have very similar relaxation magnitudes and frequencies. at low frequencies and $\varepsilon_{0}-\varepsilon_{\infty}$ values greater than 200. All studied surfactant concentrations lead to a dielectric relaxation frequency near $100 \mathrm{~Hz}$.

\subsubsection{PEG concentration}

To find the best surfactant, the effect of increasing the percentage of the polyethylene glycol (PEG) block in the triblock surfactant was also explored. With increased PEG concentration, the HLB values increases; this is summarized in Table 1, row 3. The experimental data are summarized in Table 2 and Fig. 5. These data indicate that the surfactants with low HLB (EPE0) and very high HLB (EPE4 and PEP1) lead to significantly smaller variation in the dielectric constant at low frequency, and are considered better choices as a surfactant.

\section{Discussion of Results}

The goal of the research described in this paper was to discover and characterize new emulsion dielectrics consisting of liquid crystals, surfactants and a polar solvent that at low

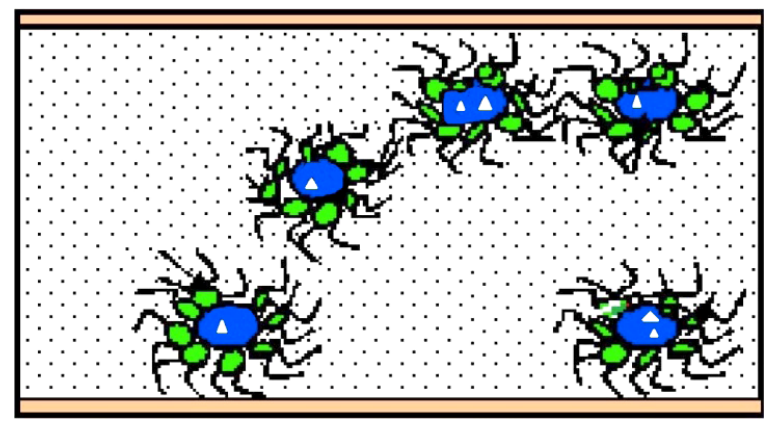

Fig. 6. (Color online) A typical system that was studied. E7 the nematic liquid crystal is the oil; NMF, N-Methylformamide is the polar solvent, a typical surfactant and ions are also shown. ( $=$ oil, blue ovals $=$ polar solvent, heads with tails $=$ surfactant, triangle $=$ ion). 


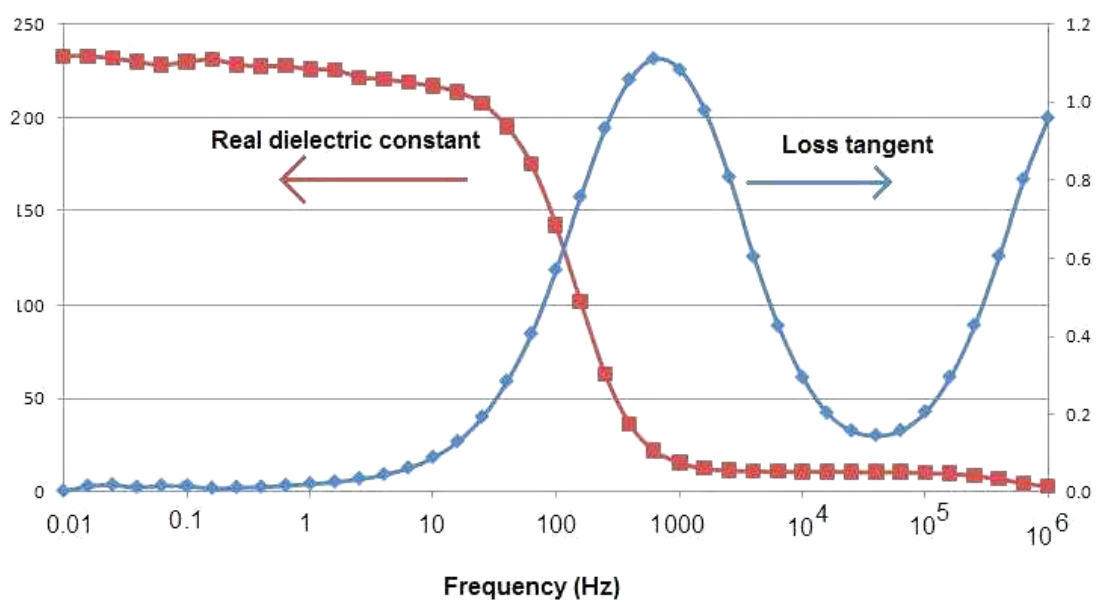

Fig. 7. The dielectric behavior of a near best case mixture. The sample contained $2 \%$ PEP1 and $98 \%$ solvent: $90.9 \%$ liquid crystal and $9.1 \%$ NMP.

frequencies $(f \rightarrow 0 \mathrm{~Hz})$ have low losses and a large real dielectric constant. The ability of organic liquid crystals to form microstructures as well as their intrinsically large real dielectric constant makes these materials excellent candidates for use in such dielectrics. However, mixtures of liquid crystal and surfactants generally produce unsatisfactory, high conductivity materials. The results presented in this paper show that this goal can be achieved in ternary mixtures, and that the losses that were observed in the individual components were reduced by using ternary mixtures. However, it was found that simply having an oil-like host, a water-like component and a surfactant did not provide a good dielectric. Some nonintuitive findings were observed. For example, replacing the solvent NMF with NMP provided a better dielectric. This is surprising since the conductivity of NMF is higher than NMP. Predictions from Maxwell-Wagner's theory would have preferred NMF.

Qualitatively it appears that "ion getters" have been formed. The liquid crystal and the polar solvent both have ions and other impurities that contribute to the significant conductivity observed at low frequencies in the pure substances. These impurities are very difficult to remove through chemical means, and many are in fact thermally generated. This suggests that the addition of surfactants and a polar solvent may improve the situation because the surfactants may form micelles that trap the ions that contribute to the conductivity at low frequencies. The results indicate that the "ion getter" effect is very effective in improving the low frequency response of these materials. A schematic drawing of such systems is shown in Fig. 6.

However, this work also indicates that all surfactants are not equally good in the present system. The right choice of surfactant was also important. For example, when the mixtures made using triblock polymeric surfactants are compared it is found that there appears to be better surfactant choices and better concentration choices. Moreover, the triblock surfactants are better than the best traditional surfactants studied.

\section{Conclusion}

The properties of ternary dielectric consisting of a liquid crystal, a triblock polymeric surfactant and a polar solvent (NMF or NMP) have been studied. The dielectric behavior of the ternary system exhibits significantly lower loss at low frequencies than the individual components. Real dielectric constants as large as 250 and loss tangents well below 0.05 are obtained in such mixtures. The loss tangent is defined by the expression

$$
\tan \delta=\frac{\omega \varepsilon^{\prime \prime}+\sigma}{\omega \varepsilon^{\prime}} .
$$

We have used the equivalent expression using the imaginary response divided by the real response. A "best case" mixture is shown in Fig. 7. At low frequencies this obtains a value near $2 * 10^{-4}$. Under similar conditions, the common plastic dielectric polyethylene obtains a real dielectric constant of 2.3 and a loss tangent of $1 * 10^{-4}$. The loss tangent peak is due to dielectric relaxation and is rather large obtaining a value near 1 . Future work will examine this phenomenon in greater detail.

The ternary mixtures are stable over periods of at least one year, and due to their liquid nature are self-healing. With further research and development these materials may be extremely useful dielectrics.

\section{Acknowledgments}

The authors wish to thank Mr. Doug Bryant for his help in sample preparation. This work was supported by the United States Office of Naval Research under contract N-000-14-051-0405.

\section{References}

${ }^{1}$ D. Ragone, Review of battery systems for electrically powered vehicles, SAE Technical Paper 680453 (1968), doi: 10.4271/ 680453. 
${ }^{2}$ E. J. Barshaw, J. White, M. J. Chait, J. B. Cornette, J. Bustamente, F. Folli, D. Biltchick, G. Borelli, G. Picci and M. Rabuffi, High energy density (HED) biaxially-oriented poly-proplyene (BOPP) capacitors for pulse power applications, IEEE Trans. Magn. 43(1), 223 (2007).

${ }^{3}$ B. Chu, X. Zhou, K. Ren, B Neese, M. Lin, Q. Wang, F. Bauer and Q. M. Zhang, A dielectric polymer with high electric energy density and fast discharge speed, Science 313, 334 (2006).

${ }^{4} \mathrm{H}$. Banno and K. Ogura, Dielectric and piezoelectric properties of a flexible composite consisting of polymer and mixed ceramic powder of PZT and $\mathrm{PbTiO}_{3}$, Ferroelectrics 95, 111 (1989).

${ }^{5}$ Y. Daben, Composite piezoelectric film made from PVDF polymer and PCM-PZT ferroelectric ceramics, Ferroelectrics 101, 291 (1990).

${ }^{6}$ Z. M. Dang, J.-B. Wu, L.-Z. Fan and C.-W. Nan, Delectric behavior of $\mathrm{Li}$ and $\mathrm{Ti}$ co-doped NiO/PVDF composites, Chem. Phys. Lett. 376, 389 (2003).

${ }^{7}$ C. Huang, Q. M. Zang and J. Su, High-dielectric-constant allpolymer percolative composites, Appl. Phys. Lett. 82(20), 3502 (2003).

${ }^{8}$ J. C. Maxwell, Electricity and Magnetism (Clarendon Press, Oxford, 1982).

${ }^{9} \mathrm{~K}$. W. Wagner, Zur theorie der unvollkommenen dielektrika, Ann. Phys. 40, 817 (1913) (in German).
${ }^{10} \mathrm{R}$. Sillars, The properties of a dielectric containing semiconducting particle of various shapes, J. Inst. Elect. Eng. 80, 378 (1937).

${ }^{11} \mathrm{~K}$. Asami, Characterization of heterogeneous systems by dielectric spectroscopy, Prog. Polym. Sci. 27, 1617 (2002).

${ }^{12} \mathrm{~S}$. Havriliak and S. Negami, A complex plane analysis of $\alpha$-dispersions in some polymer systems, J. Polym. Sci. C 14, 99 (1966).

${ }^{13}$ E. Barsoukov and J. R. Macdonald, Impedance Spectroscopy, 2nd edn. (Wiley-Interscience, Hoboken, 2005).

${ }^{14}$ P. G. de Gennes and J. Prost, The Physics of Liquid Crystals, 2nd edn. (Clarendon Press, Oxford, 1993).

${ }^{15} \mathrm{http} / / /$ kentlink.kent.edu/search/X?SEARCH=Clinton+Braganza\& searchscope $=1 \&$ SORT $=$ DX

${ }^{16}$ L. H. Sperling, Introduction to Physical Polymer Science (John Wiley, New York, 2001)

${ }^{17}$ W. Griffin, Classification of surface-active agents by "HLB", $J$. Soc. Cosmetic Chem. 1, 311 (1949).

${ }^{18} \mathrm{~W}$. Griffin, Calculation of HLB values of non-ionic surfactants, $J$. Soc. Cosmetic Chem. 5, 249 (1954).

${ }^{19} \mathrm{~S}$. H. Lui, Fractal model for the AC response of a rough interface, Phys. Rev. Lett. 55, 529 (1985).

${ }^{20}$ T. Kaplan and L. J. Gray, Effect of disorder on a fractal model for the AC response of a rough interface, Phys. Rev. B 32, 7360 (1985). 